\title{
Fatal infection in neonates of 26 weeks' gestation due to Streptococcus milleri: report of two cases
}

\author{
ROSAMOND A COX,* K CHEN,* A L COYKENDALL, $\dagger$ PAMELA WESBECHER, $\dagger$ \\ V C HERSON $\ddagger$
}

From the *Department of Pathology, Kettering and District General Hospital, Northamptonshire, the
†Department of Oral Diagnosis, School of Dental Medicine, University of Connecticut Health Centre,
Connecticut, USA, and $\ddagger$ Hartford (Connecticut) Hospital, Hartford, Connecticut, USA

SUMMARY Fatal sepsis due to a particular biotype of Streptococcus milleri occurred in two neonates born at 26 weeks' gestation. The clinical importance of this biotype in association with premature labour and neonatal sepsis is discussed.

Streptococcus milleri was first described by Guthof, who gave the name to a group of streptococci with similar biochemical and physiological properties which had been isolated from abscesses around the mouth. ${ }^{1}$ The definition of $S$ milleri as a species was further clarified by Colman and Williams, who used cell wall analysis, ${ }^{2}$ numerical classification, ${ }^{3}$ and DNA transformation ${ }^{4}$ to link taxonomically Guthof's $S$ milleri and non-haemolytic streptococci of serological groups $\mathrm{A}, \mathrm{C}, \mathrm{F}$ and $\mathrm{G}$.

Most strains are non-haemolytic, but a few are $\beta$ haemolytic and a few $\alpha$ haemolytic. One quarter to one third of all stains form the group antigen $A, C, G$ or $\mathrm{F}$, and all streptococci with the group $\mathrm{F}$ antigen have the biochemical characteristics of $S$ milleri.

Many strains grow aerobically, forming minute colonies after 24 hours, but the addition of 5-10\% carbon dioxide almost always enhances growth and colony size. Some authors have remarked on a characteristic caramel or honey smell that some isolates produce. ${ }^{56}$

Early studies of $S$ milleri were concerned with its role in dental sepsis. Its association with purulent disease in many sites was not appreciated until Parker and Ball published their study of streptococci isolated from blood cultures, cerebrospinal fluid, and internal organs. ${ }^{7}$ Later, Ball and Parker ${ }^{8}$ elaborated on the characteristics, ecology, and importance of this species. $S$ milleri has a prominent role in intra-abdominal sepsis and has been described as a cause of liver abscess, ${ }^{5}$ cholangitis, ${ }^{9}$ appendicitis, peritonitis and pelvic abscess. ${ }^{10}$

Reports of neonatal $S$ milleri infections are few. Parker ${ }^{11}$ noted one among 55 streptococcal infections

Accepted for publication 6 August 1986 in infants less than six weeks old, and there is a report of this organism causing fulminant sepsis in a neonate. ${ }^{12}$ We describe here two cases of fatal neonatal sepsis at 26 weeks' gestation due to this organism.

\section{Case reports}

\section{CASE 1 (KETTERING)}

A 30 year old married woman, gravida 4 para I, haa a cone biopsy at the age of 27 . Three years later she became pregnant shortly after the removal of an intrauterine contraceptive device. The pregnancy ended at 19 weeks with delivery of a macerated male fetus and infected placenta from which a group B $\beta$ haemolytic streptococcus was isolated. One month later she became pregnant again. Until 26 weeks this pregnancy was normal, but spontaneous rupture of membranes was followed by draining clear liquor for four days. On the fifth day liquor became meconium stained and spontaneous labour started. Ritodrine did not halt labour.

A female infant weighing $710 \mathrm{~g}$ was delivered six days after rupture of membranes. Both placenta and baby had a very offensive smell. At birth, a striking, widespread, erythematous, macular raw and weeping rash covered the infant. Her general condition was poor with gasping respirations and feeble movements (Apgar: 4 at one and four minutes). She required intermittent positive pressure ventilation for apnoeic episodes developing seven hours after birth. Parenteral penicillin and gentamicin were given as broad spectrum antibiotic cover. By 25 hours of age she had developed hypothermia $\left(28^{\circ} \mathrm{C}\right)$ and persistent acidosis, the latter partially responsive to sodium bicarbonate. After 27 hours there was anuria and a tense bulging anterior fontanelle. Intracranial haemorrhage 


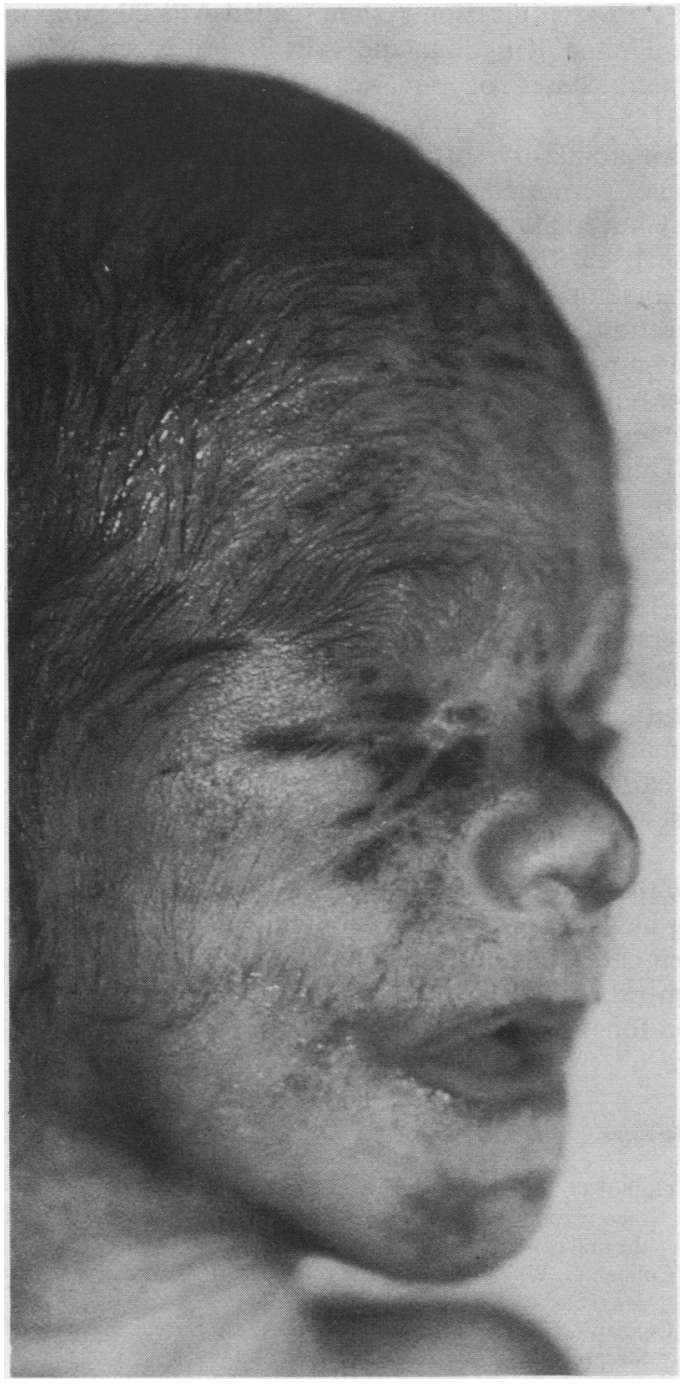

Figure Necropsy appearances of facial rash in case 1 .

was diagnosed. Cardiorespiratory support was discontinued 41 hours after birth.

Before death, swabs taken from ears, nose, throat, eyes, umbilicus and skin yielded a heavy pure growth of a non-haemolytic streptococcus, which was subsequently identified as $S$ milleri. The organism failed to grow on primary isolation on blood agar incubated in air at $37^{\circ} \mathrm{C}$. Profuse growth was obtained on blood agar incubated anaerobically (in $90 \%$ hydrogen, $10 \%$ carbon dioxide) and on chocolate agar incubated in an atmosphere of $10 \%$ carbon dioxide in a carbon dioxide incubator. All isolates had a strong caramel smell. The organism did not group with antisera of
Lancefield's groups A, B, C, D, F and G. It was identified as $S$ milleri using API 20 Strep. Necropsy samples of lung and skin also yielded the same organism in pure culture.

\section{CASE 2 (CONNECTICUT)}

A 29 year old married black woman, gravida 8, para I, spontaneous abortion 6 , had a cervical cerclage placed early in pregnancy because of cervical incompetence. The pregnancy continued uneventfully until spontaneous rupture of membranes occurred at 26 weeks' gestation. She was afebrile and the fluid appeared clear. An amniocentesis yielded clear fluid with no neutrophil polymorphs or bacteria. Betamethasone was given and the mother observed. The following day, however, the infant was delivered by caesarean section because of a falling fetal biophysical score.

The infant boy weighed $1.25 \mathrm{~kg}$, and had an Apgar score of 4 at one minute and 6 at five minutes. His estimated gestational age was 26 weeks. He was placed on mechanical ventilation because of severe hyaline membrane disease. Cultures of gastric aspirate, blood, and tracheal secretions were obtained, and he was given ampicillin and kanamycin. The infant's condition initially seemed to stabilise, but by eight hours of age he began to deteriorate. The white cell count fell from $5.8 \times 10^{9} / 1$ to $2.2 \times 10^{9} / 1$, with $16 \%$ mature neutrophil polymorphs and $16 \%$ immature neutrophil polymorphs. Chloramphenicol was added and a two volume exchange transfusion performed. Despite these measures the infant developed progressive respiratory failure with bilateral pneumothoraces and cardiovascular collapse and died at 13 hours of age.

Maternal cultures of amniotic fluid (at amniocentesis) and placenta, as well as neonatal cultures of gastric aspirate and tracheal aspirate all grew $S$ milleri. Microscopic examination of the placenta showed early chorioamnionitis.

\section{Pathology}

CASE 1

Examination showed a mildly jaundiced female fetus with a maturity of about 26 weeks and without developmental abnormality. Small strawberry coloured macules were densely scattered in a butterfly distribution on the face (figure). The rash was spread evenly over the lower abdomen and medial aspects of both thighs, with blotchy confluence over the right inferior posterior chest wall and loin regions; the loin regions had a deeper red raw appearance.

The focally aerated and consolidated lungs showed posterior congestion and scattered pleural petechiae. Histologically the lungs were irregularly expanded, 
and dilated airways contained fetal squamous debris. The lungs and all other organs examined showed no evidence of infection. The cerebral cortex was tinged yellow, and a firm, dark, slightly variegated blood clot protruded from the left lateral ventricle through the parietal lobe on to the cortical surface. Moderate amounts of blood clot enclosed the inferior surface of the cerebellar hemispheres and brain stem.

\section{CASE 2}

Examination showed a male infant, about 26 weeks of age, without congenital malformations. No rashes were noted. The lungs were consolidated and congested. Histologically the lungs were poorly aerated with early hyaline membrane formation and occasional inflammatory cells. The brain showed bilateral small subependymal haemorrhages.

\section{Discussion}

These two cases provide further examples of the pathogenic role of $S$ milleri, which caused fatal neonatal sepsis at 26 weeks' gestation. This has not, to our knowledge, been previously recorded. We suggest that in each case described here the cervix was abnormal and functionally inadequate, thus predisposing to ascending infection.

Other organisms, which may be present in the cervix (Neisseria gonorrhoeae, Chlamydia trachomatis, $U$ urealyticum, and group B streptococci), have been associated with premature labour. ${ }^{13}$ The mechanism by which this occurs remains speculative, but recent in vitro studies have shown that human amnion cells show a six-fold increase in prostaglandin E output when exposed to intact or disintegrated bacteria. ${ }^{14}$ It has been hypothesised that phospholipase $\mathbf{A}_{2}$, produced by infecting bacteria, could cause premature labour by deacylation of arachidonic acid. ${ }^{15}$

The role of $S$ milleri in the female genital tract during pregnancy and the puerperium is unknown. Poole and Wilson identified $S$ milleri in four puerperal perineal wounds (all in mixed growth) and in six patients with offensive lochia. ${ }^{16}$

The source of $S$ milleri was almost certainly the vagina. Poole and Wilson identified a biotype of $S$ milleri (raffinose + , melibiose + ) which comprised most vaginal isolates, a quarter of faecal isolates, and which was absent elsewhere. ${ }^{16}$ Ball and Parker ${ }^{8}$ noted that $S$ milleri isolates, which fermented raffinose and melibiose or mannitol, were often derived from the female genital tract. Ruoff and $\mathrm{Kunz}^{17}$ found similar streptococci in urine cultures. In both of the cases reported here the isolates conformed to this biotype. Both fermented lactose, trehalose, mannitol and raffinose, and hydrolysed aesculin. Neither was haemolytic. They produced identical reaction patterns in the API identification system (called API 20 Strep in the United Kingdom and API Rapid Strep in the United States of America); API profile number 5261551.

Inflammatory softening and rupture of the membranes permitted access to the amniotic cavity. This may have led merely to surface infection and rash in case 1, but the isolation of $S$ milleri from a necropsy lung sample is presumptive evidence of systemic infection. The absence of obvious histological inflammatory changes in either of these cases does not necessarily exclude systemic infection as the immune system at 26 weeks is exceedingly immature.

Thus infection with $S$ milleri in utero led to the birth of two infants who were compromised both by extreme prematurity and systemic infection. The incidence of neonatal sepsis caused by non-haemolytic or "viridans" streptococci seems to be increasing, at least in North America. ${ }^{18} 19$ Because this biotype of $S$ milleri is usually not haemolytic, it is probably identified simply as a non-haemolytic streptococcus in many laboratories. In the light of our two cases and an earlier report, ${ }^{12}$ this species should be suspected in cases of neonatal sepsis and septic abortion.

We thank Mr RJ Smith and Dr EH Smith for allowing us to report the clinical details of patients undeg their care in Kettering. We also thank Dr G Colman Central Public Health Laboratory, Colindale, Lon don for the initial identification of the Kettering iso late.

\section{References}

1 Guthof O. Pathogenic strains of Streptococcus viridans: streptococci found in dental abscesses and infiltrates in the region of the oral cavity. Zentrabl Bakteriol Parasitenk 1956;166:553-64.

2 Colman G, Williams REO. The cell walls of streptococci. J Gen Microbiol 1965;41:375-87.

3 Colman G. The application of computers to the classification of Streptococci. J Gen Microbiol 1968;50:149-58.

4 Colman G. Transformation of viridans-like streptococci. J Gen Microbiol 1969;57:247-55.

5 Moore-Gillon JC, Eykyn S, Phillips I. Microbiology of pyogenic liver abscess. Br Med J 1981;283:819-21.

6 Shlaes DM, Lerner PI, Wolinsky E, Gopalakrishna KV. Infections due to Lancefield group $F$ and related streptococci (S milleri, S angiosus). Medicine 1981;60:197-207.

7 Parker MT, Ball LC. Streptococci and aerococci associated with systemic infection in man. $J$ Med Microbiol 1976;9:275-302.

8 Ball LC, Parker MT. The cultural and biochemical characters of Streptococcus milleri strains isolated from human sources. $J$ Hyg (Camb) 1979;82:63-78.

9 Murray HW, Gross KC, Masur H, Roberts RB. Serious infections caused by Streptococcus milleri. Am J Med 1978; 64:759-64.

10 Poole PM, Wilson G. Streptococcus milleri in the appendix. $J$ Clin Pathol 1977;30:937-42.

11 Parker MT. Neonatal streptococcal infections. Postgrad Med J 1977;53:598-606.

12 Spencer RC, Nanayakarra CS, Coup AJ. Fulminant neonatal sepsis due to Streptococcus milleri. J Infect 1982;4:88-9. 
13 Eschenbach DA, Gravett MG, Chen KCS, Hoyme UB, Holmes KK. Bacterial vaginosis during pregnancy. An association with prematurity and postpartum complications. In: Mardh PA, Taylor-Robinson J, eds. Bacterial vaginosis. Stockholm: Almquist and Wiskell International, 1984:213-22.

14 Lamont RF, Rose M, Elder MG. Effect of bacterial products on prostaglandin $\mathrm{E}$ production by amnion cells. Lancet 1985;ii:1331-3.

15 Bejar R, Curbelo V, Davis C, Gluck L. Premature labour II. Bacterial sources of phospholipase. Obstet Gynecol 1981; 57:479-82.

16 Poole PM, Wilson G. Occurrence and cultural features of Streptococcus milleri in various body sites. J Clin Pathol 1979;32:764-8.
17 Ruoff KL, Kunz LJ. Identification of viridans streptococci isolated from clinical specimens. $J$ Clin Microbiol 1982;15:920-5.

18 Broughton RA, Krafka R, Baker CJ. Non-group D alphahaemolytic streptococci: new neonatal pathogens. $J$ Pediatr 1981;99:450-4.

19 Spigelblatt L, Saintonge J, Chicoine R, Lavieriore M. Changing pattern of neonatal streptococcal septicaemia. Pediatr Infect Dis 1985;4:56-8.

Requests for reprints to: Dr Rosamond A Cox, Department of Pathology, Kettering and District General Hospital, Kettering, Northants NN16 8UZ, England. 